\title{
Enhanced Reverse Saturable Absorption in Substituted Twistacenes from Visible to Near-Infrared: Modulation of Terminal Twisted ח-Conjugated Units
}

Jidong Jia, Xingzhi Wu, Yu Fang, Junyi Yang, Yanbing Han, Jinchong Xiao*, Xueru Zhang*, Yuxiao Wang, and Yinglin Song*

\section{Supporting Information}

Synthesis of $\mathbf{H 4}$

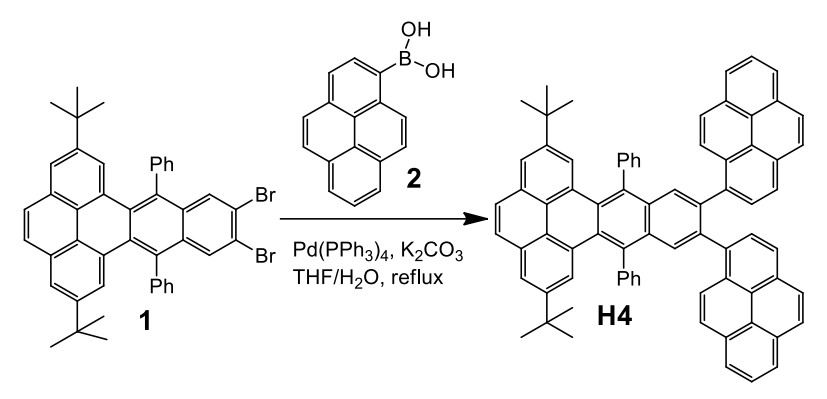

Scheme S1. The synthetic route of $\mathbf{H 4}$.

A mixture of pyren-1-ylboronic acid (177 mg, $0.72 \mathrm{mmol}), 11,12$-dibromo-2,7-di-tertbutyl-9,14-diphenyldibenzo[de,qr]tetracene (265 mg, $0.36 \mathrm{mmol}), \operatorname{Pd}\left(\mathrm{PPh}_{3}\right)_{4}(14 \mathrm{mg}$, $0.012 \mathrm{mmol})$ and $\mathrm{K}_{2} \mathrm{CO}_{3}(50 \mathrm{mg}, 0.36 \mathrm{mmol})$ was stirred in THF and water (v/v, 20 $\mathrm{mL}: 10 \mathrm{~mL}$ ) at $80^{\circ} \mathrm{C}$ under nitrogen atmosphere. After $16 \mathrm{~h}$, the solution was cooled down to room temperature. After removing THF, brine was added. The resulting solution was extracted with dichloromethane $(30 \mathrm{~mL} \times 3)$. The organic layer was dried over $\mathrm{Na}_{2} \mathrm{SO}_{4}$ and evaporated in vacuo. The crude product was purified through column chromatography on silica gel by using $\mathrm{PE}$ and $\mathrm{CH}_{2} \mathrm{Cl}_{2}$ (v/v, 8:1) to afford a yellow solid (205 mg, 59\%). ${ }^{1} \mathrm{H}$ NMR (600 MHz, $\left.296 \mathrm{~K}, \mathrm{CDCl}_{3}\right): \delta=8.66(\mathrm{~d}, J=9.6 \mathrm{~Hz}, 1 \mathrm{H}), 8.36(\mathrm{~d}$, $J=9.6 \mathrm{~Hz}, 1 \mathrm{H}), 8.29-8.24(\mathrm{~m}, 7 \mathrm{H}), 8.21(\mathrm{~d}, J=7.8 \mathrm{~Hz}, 1 \mathrm{H}), 8.15(\mathrm{~d}, J=7.8 \mathrm{~Hz}, 1 \mathrm{H})$, 
$8.05(\mathrm{t}, 1 \mathrm{H}), 8.00(\mathrm{~d}, J=7.8 \mathrm{~Hz}, 1 \mathrm{H}), 7.96-7.81(\mathrm{~m}, 11 \mathrm{H}), 7.76(\mathrm{t}, 2 \mathrm{H}), 7.64(\mathrm{t}, 1 \mathrm{H}), 7.51-$ $7.48(\mathrm{~m}, 3 \mathrm{H}), 7.45(\mathrm{~d}, J=7.2 \mathrm{~Hz}, 1 \mathrm{H}), 7.40(\mathrm{~d}, J=7.2 \mathrm{~Hz}, 1 \mathrm{H}), 7.32-7.27(\mathrm{~m}, 3 \mathrm{H}), 7.24$ (s, 1H), 1.18 (s, 18H). ${ }^{13} \mathrm{C}$ NMR (150 MHz, $\left.296 \mathrm{~K}, \mathrm{CDCl}_{3}\right): \delta=147.4,142.34,142.29$, $138.9,138.7,137.0,136.9,136.2,133.0,132.6,132.4,131.8,131.4,131.2,131.1,130.9$, $130.8,130.7,130.62,130.60,130.4,130.1,129.9,129.70,129.67,129.5,129.4,129.0$, $128.9,128.8,128.1,127.8,127.6,127.44,127.37,127.2,127.1,127.0,126.8,125.9$, $125.8,125.7,125.6,125.0,124.9,124.80,124.76,124.6,124.5,124.0,123.98,123.8$, 122.4, 34.8, 31.4. HR-MS (MS, m/z), calc. for $\mathrm{C}_{76} \mathrm{H}_{54}$ : 966.4226 ; found 966.4209 . 


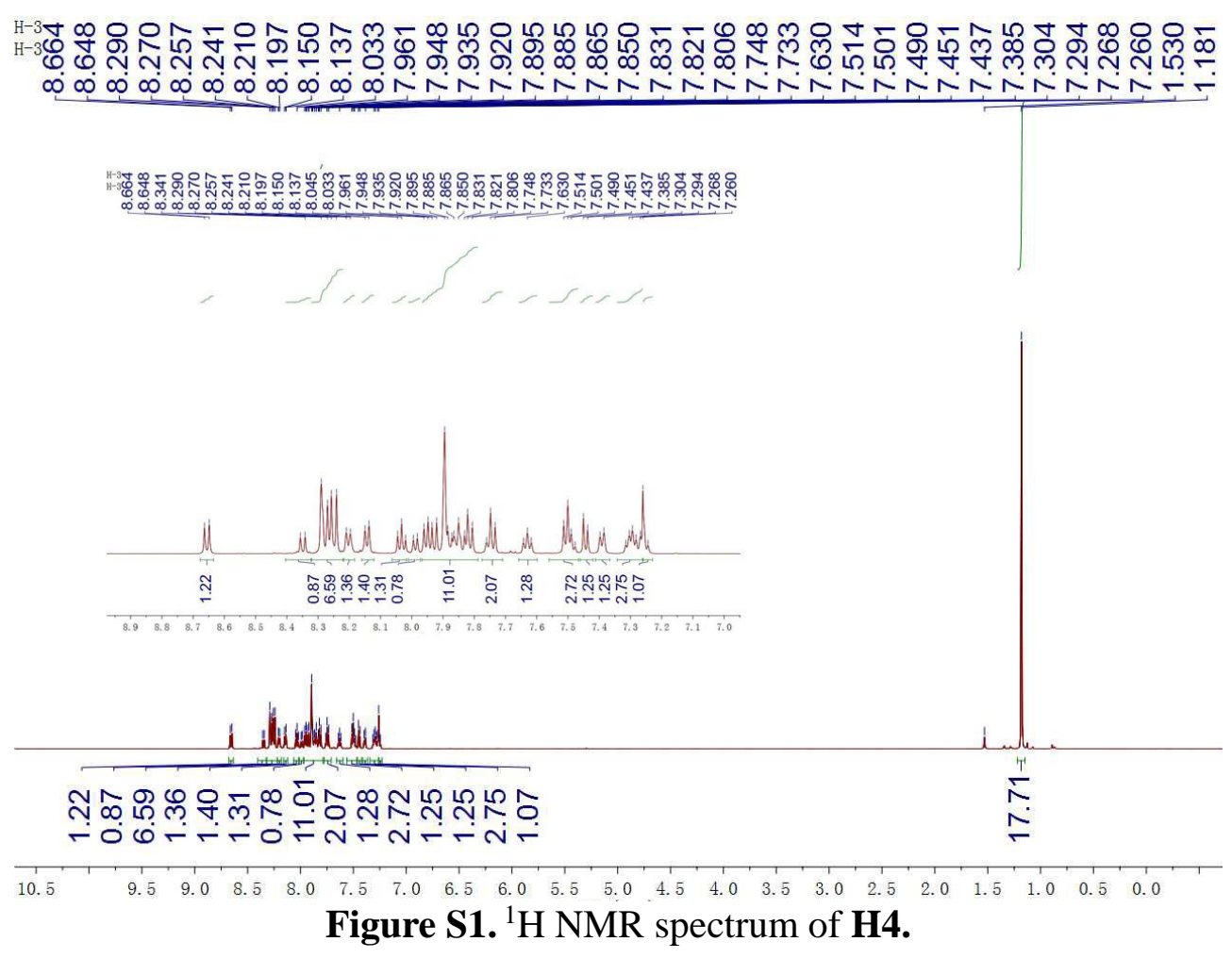

H-3อ

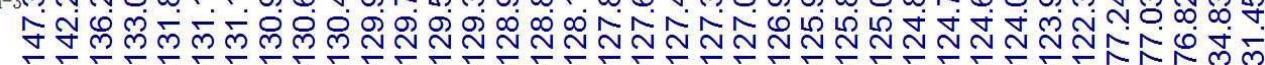

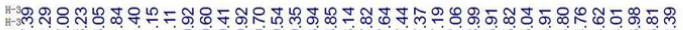

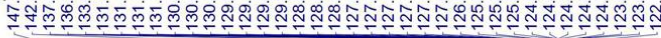

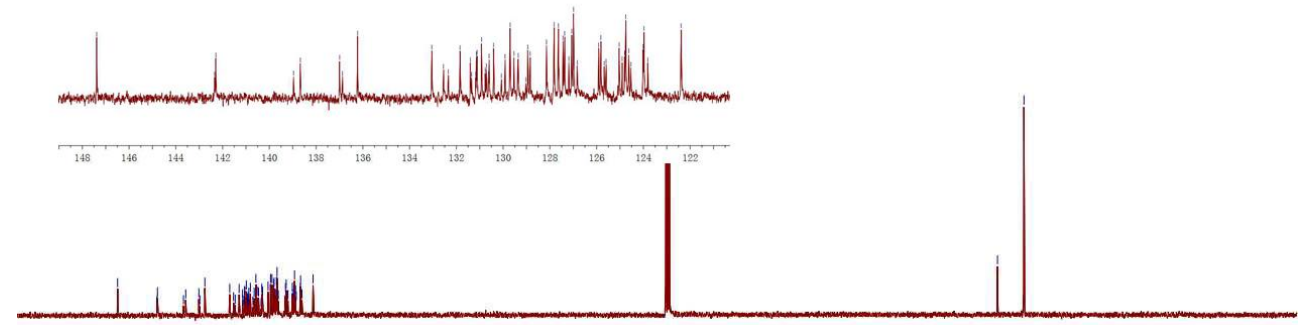

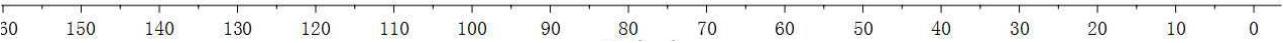

Figure S2. ${ }^{13} \mathrm{C}$ NMR spectrum of $\mathbf{H 4}$. 

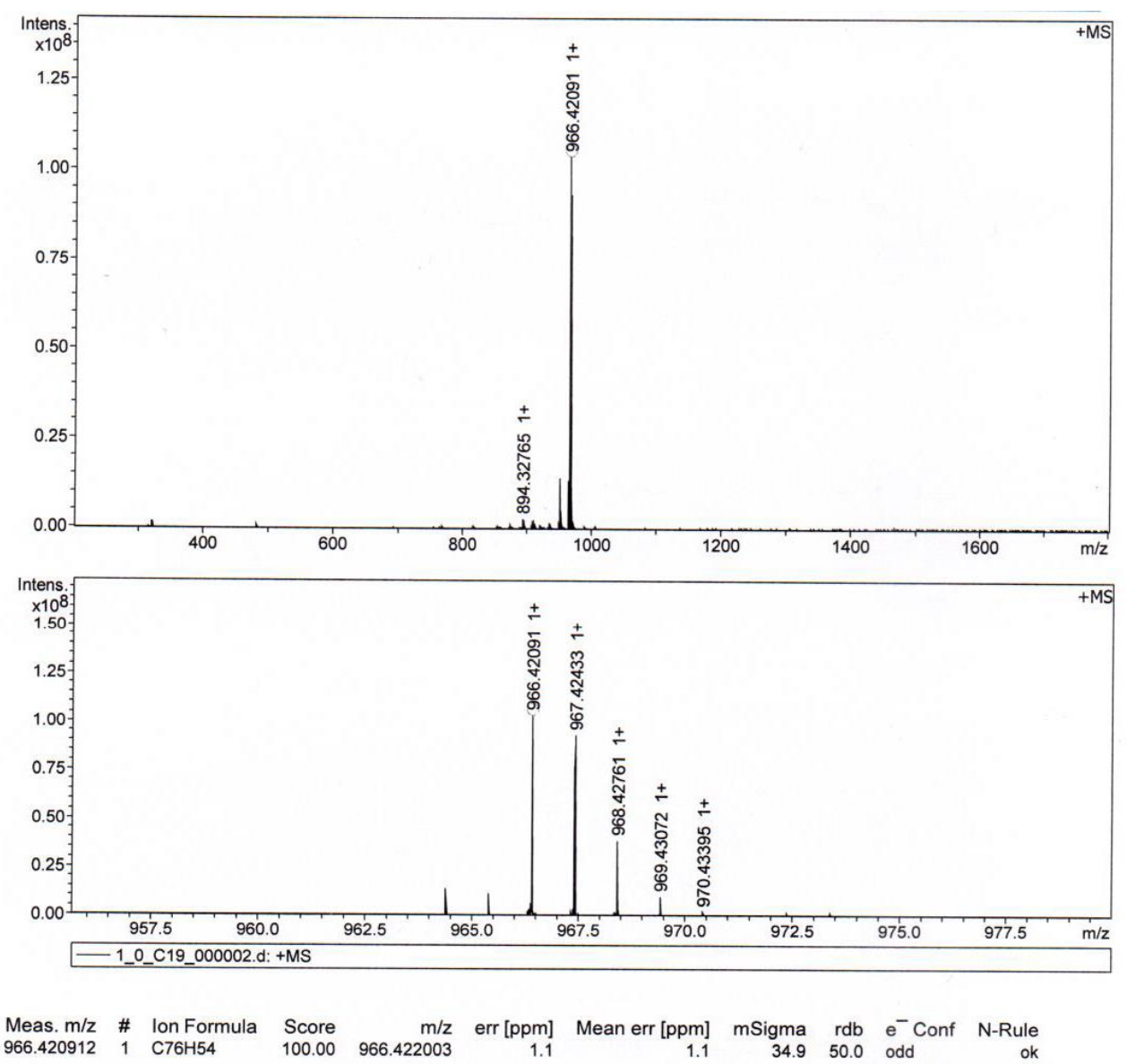

Figure S3. HR-MS spectrum of $\mathbf{H 4}$.

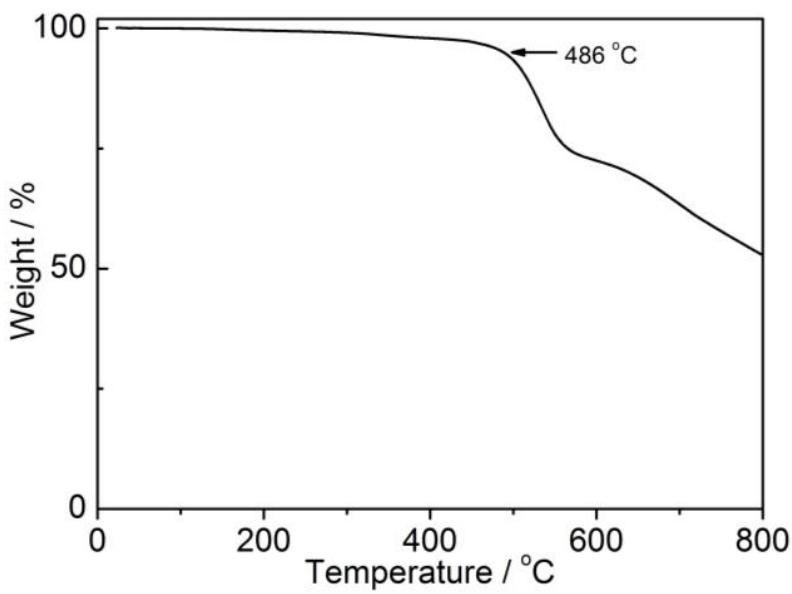

Figure S4. TGA datum of $\mathbf{H 4}$ under nitrogen atmosphere. 


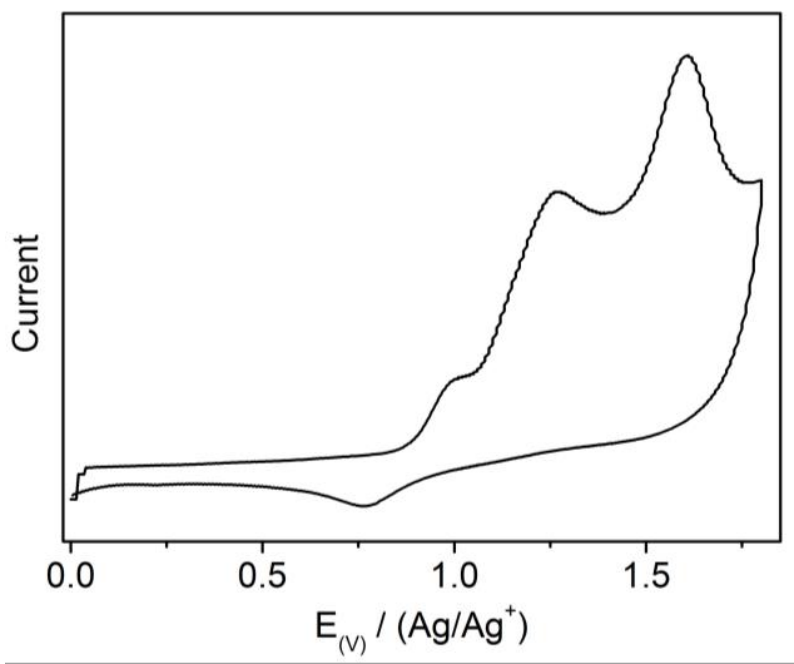

Figure S5. $\mathrm{CV}$ spectrum of $\mathbf{H 4}$ in $0.1 \mathrm{M} \mathrm{TBAPF} / \mathrm{CH}_{2} \mathrm{Cl}_{2}$ with ferrocene as the internal standard. 